\title{
Structure and Bonding in lonized Water Clusters
}

\author{
Hainam Do and Nicholas A. Besley* \\ School of Chemistry, University of Nottingham, University Park, Nottingham, NG7 2RD, UK. \\ E-mail: nick.besley@nottingham.ac.uk
}

*To whom correspondence should be addressed 


\begin{abstract}
The structure and bonding in ionized water clusters, $\left(\mathrm{H}_{2} \mathrm{O}\right)_{n}^{+}(n=3-9)$, has been studied using the basin hopping search algorithm in combination with quantum chemical calculations. Initially candidate low energy isomers are generated using basin hopping in conjunction with density functional theory. Subsequently, the structures and energies are refined using second order Møller-Plesset perturbation theory and coupled cluster theory, respectively. The lowest energy isomers are found to involve proton transfer to give $\mathrm{H}_{3} \mathrm{O}^{+}$and a $\mathrm{OH}$ radical, which are more stable than isomers containing the hemibonded hydrazine-like fragment $\left(\mathrm{H}_{2} \mathrm{O}-\mathrm{OH}_{2}\right)$, with the calculated infrared spectra consistent with experimental data. For $\left(\mathrm{H}_{2} \mathrm{O}\right)_{9}^{+}$the observation of a new structural motif comprising proton transfer to form $\mathrm{H}_{3} \mathrm{O}^{+}$and $\mathrm{OH}$, but with the $\mathrm{OH}$ radical involved in hemibonding to another water molecule is discussed.
\end{abstract}

keywords: clusters, basin hopping, hemibonding 


\section{Introduction}

Characterizing the structure and properties of water clusters has been the subject of an extensive research effort for many years. Through such studies it is possible to explore the nature and evolution of the hydrogen bonding network as the number of water molecules is increased, in an effort to understand the complex hydrogen bonding network that exists in liquid water. ${ }^{1-9}$ The structure of water clusters after exposure to ionizing radiation is also important in a number of fields spanning chemistry, biology and physics, for example in the neutron irradiation of cooling water in a nuclear atomic plant, the damage of living cells by radiation and in the photoreaction of water in the atmosphere. ${ }^{2,10-12}$ Furthermore, the $\mathrm{OH}$ radicals that can be produced on ionization have been associated with cell death. ${ }^{13}$

Several groups have studied ground state cationic water clusters $\left(\mathrm{H}_{2} \mathrm{O}\right)_{n}^{+}$in experiment. ${ }^{14-17}$ Studying these clusters in experiment is challenging since the unprotonated cluster ions are formed in a highly excited vibrational state. ${ }^{18,19}$ This leads to fragmentation of the water clusters on an ultrafast time scale to give rise to protonated species. ${ }^{20}$ However, several studies have measured unprotonated cationic water clusters, ${ }^{14-17}$ and recently the infrared spectroscopy of size selected unprotonated cationic water clusters was reported. ${ }^{16}$ In these clusters, there is the possibility of several distinct structural motifs, which are illustrated in Figure $1 .{ }^{11,17}$ The first possibility a has a hydrazine-like core $\left(\mathrm{H}_{2} \mathrm{O}-\mathrm{OH}_{2}\right)$ with a hemibond between the two oxygen atoms. The nature of singly charged hemibonded systems has been studied by Gill and Radom, where a three electron hemibonded system is described as one in which a bonding $\sigma$ molecular orbital between two atoms in a molecule is doubly occupied while the complementary antibonding $\sigma^{*}$ orbital is singly occupied. ${ }^{21}$ These orbitals are shown for the $\left(\mathrm{H}_{2} \mathrm{O}\right)_{3}^{+}$cluster in Figure 2. Evidence of the hemibonding is demonstrated by the spin density (shown in Figure 1), which for a is distributed equally on the two oxygen atoms of the hydrazine-like core. In the remaining structures, proton transfer has occurred to give a $\mathrm{H}_{3} \mathrm{O}^{+}$ion and $\mathrm{OH}$ radical. In $\mathbf{b}$ the ion and the radical are hydrogen bonded to each other to give an ion-radical pair, while in $\mathbf{c}$ there is a central core of $\mathrm{H}_{3} \mathrm{O}^{+}$with the $\mathrm{OH}$ radical 
is not directly hydrogen bonded to $\mathrm{H}_{3} \mathrm{O}^{+}$. For these structures, the spin density is localized on the $\mathrm{OH}$ radical. For larger clusters there is a further possibility labelled $\mathbf{d}$. This structure involves the formation of a $\mathrm{H}_{3} \mathrm{O}^{+}$ion and $\mathrm{OH}$ radical but the $\mathrm{OH}$ radical forms a hemibond with a further water molecule. In the literature, there is not a clear consensus over the most favored structural motif with studies predicting both hydrazine-like ${ }^{22}$ and radical-ion pair type structures. ${ }^{11,17}$ Recently, Mizuse et al. have studied cationic water clusters using infrared (IR) spectroscopy in combination with theoretical calculations and found that the $\mathrm{H}_{3} \mathrm{O}^{+}-\mathrm{OH}$ ion-radical pair dominates, with motif c favored for $\mathrm{n} \geq 5$. $^{16}$

Several theoretical studies have addressed the energetic and structural properties of cationic water clusters. ${ }^{18,19,21,23-28}$ However, in a similar manner to experimental work on these clusters, there has also been some disagreement between theoretical studies. Early work using density functional theory (DFT) predicted that structures containing the hydrazine-like core were favored relative to the dissociated (or proton-transfer) structures. ${ }^{18,19}$ Later studies using wavefunction based approaches, such as coupled cluster theory and Møller-Plesset peturbation theory, have shown that for the water dimer cation the $\mathrm{H}_{3} \mathrm{O}^{+}-\mathrm{OH}$ ion-radical pair has the lowest energy. ${ }^{23,27,28}$ This is illustrated clearly by a recent study on the water trimer cation, where it was also shown that DFT with carefully chosen exchange-correlation functionals could predict the relative energies of the different isomers correctly. ${ }^{24}$ To determine low energy structures for the cationic water clusters, $\left(\mathrm{H}_{2} \mathrm{O}\right)_{n}^{+}$ $(n=3-9)$ requires an extensive search of possible configurations combined with an accurate method for determining the relative energies of the clusters. The basin hopping $(\mathrm{BH})^{29}$ algorithm is a combination of the Metropolis Monte Carlo (MC) sampling technique and a gradient-based local search method, which has the effect of sampling the energy basins instead of sampling configuration space. The BH approach in conjunction with different potential functions has been used successfully to determine the minimum energy structures of the related system of protonated water clusters. ${ }^{30,31}$ In this work, the structure of candidate low energy structures was determined with DFT in conjunction with our recent implementation ${ }^{9}$ of the $\mathrm{BH}$ algorithm within the Q-Chem 
software package. ${ }^{32}$ Subsequently, the structures were refined using second order Møller-Plesset perturbation theory (MP2) and relative energies determined in coupled cluster theory calculations.

\section{Computational Details}

For each cluster, ten separate BH searches, consisting of $1000 \mathrm{MC}$ steps at $300 \mathrm{~K}$ starting from different randomly generated configurations of molecules were performed. At each MC step, all molecules are translated and rotated twice. The nature of the transformed surface allows for relatively large step sizes, and the maximum displacement in the translation move is set to $2.5 \AA$ and the maximum rotation set to $\pi / 2$. The atoms are prevented from getting closer than $1.5 \AA$ to avoid overlapping which can lead to the self-consistent field (SCF) calculation failing to converge. Thus, any move that brings at least one atom pair within $1.5 \AA$ is discarded, and when a molecule leaves the box, it is placed back in the opposite side of the box. If the MC moves are rejected ten times, a jumping procedure ${ }^{33}$ consisting of seven random walks in the configurational space will take place allowing the system to escape from the local minimum.

The energies and gradients were computed using the B3LYP exchange-correlation functional ${ }^{34,35}$ in conjunction with $6-31+\mathrm{G}^{*}$ basis set within an unrestricted Kohn-Sham formalism. For cationic water clusters, we find that incorporating dispersion (via the empirical dispersion correction of Grimme $^{36}$ ) does not change the nature of the low lying isomers except that the hydrazine-like core isomers are stabilized more relative to the radical-ion isomers. To speed up the calculations, the integration grid is reduced from the default SG1 grid to a 25 point Euler-Maclaurin radial grid and 50 point Lebedev angular grid, and for the convergence in the geometry optimisation, the energy convergence criteria is loosened to $10^{-5}$ a.u., the root mean squared gradient to fall below $2 \times 10^{-3}$ a.u. and the convergence criterion for displacement is set to $2 \times 10^{-3}$ a.u. This provides a good balance between the quality of the evaluated energy and efficiency, particularly since this 
step is to produce candidate structures that are subject to further refinement of the structures and energies. Previous studies have shown that DFT calculations do not predict the relative stability of the hemibonded and ion-radical isomers correctly. ${ }^{24}$ and the low energy candidate structures from the BH search were re-optimized at the MP2/6-311++G** level and the relative energies of the clusters determined from $\operatorname{CCSD}(\mathrm{T})$ calculations. The $\operatorname{CCSD}(\mathrm{T})$ calculations used the aug-ccpVTZ basis set was used for $n \leq 6$ and aug-cc-pVDZ for the larger clusters and were performed using the MOLPRO software. ${ }^{37}$ Harmonic vibrational frequencies were also computed to provide an estimate of the zero point energy and to allow a direct comparison with experimental data. To avoid computationally expensive frequency evaluations using MP2, the frequencies were evaluated with B3LYP with the empirical dispersion correction of Grimme ${ }^{36}$ in conjunction with the $6-311++\mathrm{G}^{* *}$ basis set. The standard values of $s_{6}=1.05$ and $d=20.0$ for B3LYP were used. ${ }^{36}$ Prior to the frequency calculations the structure of the clusters were re-optimized at this level of theory but this did not result in a structural change to give a cluster corresponding to a different local minimum. IR spectra were generated by representing the vibrational bands as gaussian functions with a full width at half maximum of $10 \mathrm{~cm}^{-1}$ and $30 \mathrm{~cm}^{-1}$ for bands above and below 3550 $\mathrm{cm}^{-1}$, respectively, following scaling of the computed frequencies by $0.9679 .{ }^{38}$ The band widths were chosen to provide a good representation of the reported experimental data. ${ }^{16}$

\section{Results and Discussion}

Figures 3 and 4 show the structures and relative energies of the low energy isomers of the $\left(\mathrm{H}_{2} \mathrm{O}\right)_{3-9}^{+}$ clusters, with relative energies incorporating zero point energies shown in parenthesis. For $n=3$, the lowest energy structure has structural motif $\mathbf{b}$ with $\mathrm{H}_{3} \mathrm{O}^{+}$bonded to a water molecule and $\mathrm{OH}$ radical. There will clearly be some relationship between the structures of protonated clusters and cationic clusters, and this cluster can also be viewed as a $\mathrm{OH}$ radical bonded to a Zundel cation. The lowest energy isomer with the hydrazine-like core is $39 \mathrm{~kJ} / \mathrm{mol}$ higher in energy, and has a water molecule hydrogen bonded to both water molecules of the hydrazine-like core, consistent with 
earlier work. ${ }^{24}$ Inclusion of zero point energy leads to an increase in the relative stability of the ion-radical isomer by $12 \mathrm{~kJ} / \mathrm{mol}$. A further isomer with a water molecule hydrogen bonded to just one of the water molecules of the hydrazine-like core is found higher in energy. On optimisation with MP2 this structure reverts to the more stable hemibonded structure, and the structure shown corresponds to the DFT optimised structure. This is also observed for the hemibonded structures of some of the larger clusters. We note that the relative energies of these isomers predicted by the B3LYP calculations is different, with the lowest energy isomer predicted to have the hydrazine-like core. This is a consequence of the DFT calculations over estimating the stability of the oxygenoxygen hemibond. ${ }^{24}$

Figure 5 shows the computed IR spectra for the two lowest energy isomers for frequencies $>$ $3400 \mathrm{~cm}^{-1}$. Also shown are spectra measured in experiment from the work of Mizuse et al. ${ }^{16}$ The vibrational modes that occur in this region of the spectrum correspond to the $\mathrm{O}-\mathrm{H}$ stretching modes of the water molecules. For an accurate simulation of experiment it is necessary to compute both accurate frequencies and the relative intensities of the bands. The DFT frequency calculations used here should provide qualitatively accurate relative intensities of the bands. In the spectrum for the ion-radical structure, the $\mathrm{O}-\mathrm{H}$ stretch in the $\mathrm{OH}$ radical is also evident as a weak band at 3550 $\mathrm{cm}^{-1}$. In the hemibonded isomer the $\mathrm{O}-\mathrm{H}$ stretching modes of the hemibonded water molecules are shifted to lower frequency and lie between 3100 and $3400 \mathrm{~cm}^{-1}$ and are not shown. The experimental spectrum has a weak band at $3530 \mathrm{~cm}^{-1}$, a strong band at $3624 \mathrm{~cm}^{-1}$ and a moderately intense band at $3710 \mathrm{~cm}^{-1}$. The computed spectrum for the ion-radical isomer has bands at 3550 $\mathrm{cm}^{-1}, 3665 \mathrm{~cm}^{-1}$ and $3760 \mathrm{~cm}^{-1}$ with a similar pattern of intensities. This is more consistent with experiment than the spectrum for hemibonded isomer which has three bands but with the lowest frequency band being the most intense.

$\left(\mathrm{H}_{2} \mathrm{O}\right)_{4}^{+}$has several relatively low energy ion-radical isomers. The lowest energy structure has two water molecules and the $\mathrm{OH}$ radical hydrogen bonded to $\mathrm{H}_{3} \mathrm{O}^{+}$in an approximately trigo- 
nal arrangement. This is similar to the lowest energy structure of the analogous protonated water cluster, which also has a trigonal arrangement with $\mathrm{H}_{3} \mathrm{O}^{+}$at the centre. ${ }^{30}$ Two further isomers in which $\mathrm{H}_{3} \mathrm{O}^{+}$is hydrogen bonded to one water molecule and the $\mathrm{OH}$ radical that lie $8-10 \mathrm{~kJ} / \mathrm{mol}$ higher in energy are also identified, and the lowest energy isomer with the hydrazine-like core is predicted to be significantly higher in energy. The computed IR spectrum for the lowest energy ion-radical isomer is in good agreement with the experimental spectrum, and provides a closer match to experiment than the next lowest energy isomer. In both spectra, the band arising from the $\mathrm{OH}$ stretch in the radical is evident and the importance of this band in indicating the presence of the ion-radical isomers has been identified in earlier work. ${ }^{16}$ For $\left(\mathrm{H}_{2} \mathrm{O}\right)_{5}^{+}$, there is the emergence of structural motif $\mathbf{c}$ with a separated ion-radical pair, which is found to have the lowest energy. This structure can also be viewed as a $\mathrm{OH}$ radical bonded to an Eigen cation. Two isomers with the ion-radical pair (b) are predicted with relative energies of $+4 \mathrm{~kJ} / \mathrm{mol}$ and $+9 \mathrm{~kJ} / \mathrm{mol}$. Again for this cluster, the lowest energy hydrazine-like isomer is over $40 \mathrm{~kJ} / \mathrm{mol}$ higher in energy. The computed IR spectrum for the lowest ion-radical cluster provides an excellent match for the experimental spectrum

The lowest energy isomer of $\left(\mathrm{H}_{2} \mathrm{O}\right)_{6}^{+}$cluster has structural type $\mathbf{c}$, with $\mathrm{H}_{3} \mathrm{O}^{+}$bonded to two water molecules. Two further low lying isomers with structural type $\mathbf{c}$ but with slightly different arrangements of the water molecules are also found. These isomers are predicted to be within 2 $\mathrm{kJ} / \mathrm{mol}$ higher in energy. There are significant differences between the structures of the cationic and neutral clusters. For $n=6$, neutral clusters form cages, while the cationic clusters still favor structures with chains and rings of water molecules. The predicted IR spectrum for both of these isomers agree well with experiment and it is difficult to differentiate between them based upon the IR spectra. However, there is a small peak at $3580 \mathrm{~cm}^{-1}$ which is evident in experiment and the spectrum for the lowest energy isomer, but is absent in the spectrum for the higher energy isomer. For the $\left(\mathrm{H}_{2} \mathrm{O}\right)_{n=3-6}^{+}$clusters, the findings in this study are consistent with the results of Mizuse et al. ${ }^{16}$ 
Figure 4 shows structures and relative energies for the $\left(\mathrm{H}_{2} \mathrm{O}\right)_{7-9}^{+}$clusters. For $n=7$ there is a significant change, with the the presence of cage-like structures predicted among the low energy isomers. Although, once zero point energies are accounted for the lowest energy isomer has an approximately trigonal planar structure with a separated ion-radical pair. While the computed energy difference is less that $1 \mathrm{~kJ} / \mathrm{mol}$, the computed IR spectra for the lowest energy ion-radical isomer is more consistent with the reported experimental spectrum, although the weak band observed just above $3600 \mathrm{~cm}^{-1}$ in the experiment is predicted to lie at a lower frequency. The relative energy of the lowest hemibonded structure remains high but the calculations to show that this energy difference decreases as the size of the clusters increase.

As the clusters become larger, identifying the lowest energy isomer becomes more challenging. $\left(\mathrm{H}_{2} \mathrm{O}\right)_{8}^{+}$is the smallest cluster that is predicted to have a cage-like lowest energy structure. The lowest energy structure is predicted to have structural type $\mathbf{c}$, with a separated ion-radical pair. The computed IR spectrum for this cluster is very similar to the experimental spectrum indicating that the correct structure has been identified. There is more ambiguity regarding the precise nature of the lowest energy structure for $\left(\mathrm{H}_{2} \mathrm{O}\right)_{9}^{+}$. The two lowest energy isomers are very close in energy. The lowest energy structure has an ion-radical pair, while the second lowest energy isomer has a separated ion-radical pair. The computed IR spectra of either of these isomers does not provide a good match with the experimental spectrum. Although a Boltzmann weighted spectrum, at a temperature of $180 \mathrm{~K}$ in line with experiment, ${ }^{16}$ for the two lowest isomers is in better, although not perfect, agreement with experiment. Closer observation of the lowest isomer shows the possibility of hemibonding between the $\mathrm{OH}$ radical and the water molecule below it, as illustrated in Figure 4. The predicted IR band at $3635 \mathrm{~cm}^{-1}$ corresponds to the stretching modes of this water molecule and $\mathrm{OH}$ radical and is shifted to lower wavenumber than the stretching modes of the remaining water molecules because of the hemibonding. The experimental spectrum has a band at $3650 \mathrm{~cm}^{-1}$ which could possibly correspond to this. Hemibonding between water and a $\mathrm{OH}$ radical has been 
considered previously, and calculations suggest that the major peak in the ultraviolet absorption spectrum of an $\mathrm{OH}$ radical in liquid water arises from $\mathrm{OH}$ hemibonded to water. ${ }^{26,39}$ The answer to whether hemibonding does exist between the $\mathrm{OH}$ radical and water molecule highlights the difficulty of modelling these systems. For the cluster considered here, the answer depends on the type of calculation. The B3LYP+D calculations predict the $\mathrm{H}_{2} \mathrm{O}-\mathrm{OH}$ bond length to be $2.37 \AA$ and there is significant spin density on the oxygen atom of the water molecule indicating the presence of hemibonding. However, at the MP2 level the $\mathrm{H}_{2} \mathrm{O}-\mathrm{OH}$ bond length is significantly greater at $2.98 \AA$ with only a small delocalisation of the spin density from the radical to the water molecule, suggesting that hemibonding is not important.

\section{Conclusions}

The lowest energy isomers for $\left(\mathrm{H}_{2} \mathrm{O}\right)_{n}^{+}(n=3-9)$ have been determined in a hierarchical approach using a combination of the BH search algorithm with DFT, MP2 and CCSD(T). The computational cost of performing the BH with DFT is large and this cost could be reduced using cheaper, more empirical methods for the search. However, one of the advantages of using DFT is its wide applicability and does not require the development of potentials for each system that is studied. For $n=3-8$, the computed IR spectra are consistent with experimental measurements providing evidence that the correct lowest energy isomers have been identified. The lowest energy structures are predicted to conform to the ion-radical structural forms $\mathbf{b}$ and $\mathbf{c}$, and the lowest energy isomers based upon the $\left(\mathrm{H}_{2} \mathrm{O}-\mathrm{OH}_{2}\right)$ hemibonded structure are predicted to significantly higher in energy. For $\left(\mathrm{H}_{2} \mathrm{O}\right)_{9}^{+}$there is more ambiguity regarding the precise nature of the lowest energy structure, with a Boltzmann weighted spectrum for the two lowest isomers required to give approximate agreement with experiment. This cluster also highlights the possibility of hemibonding occurring between the $\mathrm{OH}$ radical and water molecule. Using DFT, this type of hemibonding is predicted to be significant while at the MP2 level this bonding is less evident. 


\section{Acknowledgments}

We thank the University of Nottingham High Performance Computing facility for providing computer recourses and the Engineering and Physical Sciences Research Council (EPSRC) for funding (Grant No. EP/I012303). The authors would like to thank Professor Asuka Fujii (Graduate School of Science, Tohoku University, Japan) for providing his experimental data in electronic form.

\section{Supporting Information Available}

Cartesian coordinates of the optimised structures and the computed infrared frequencies and intensities along with the full citations for references 13, 32 and 37 are provided in the Supporting Information. This material is available free of charge via the Internet at http://pubs.acs.org

\section{References}

(1) Tsai, C. J.; Jordan; K. D. Theoretical Study of Small Water Clusters: Low-energy Fused Cubic Structures for $\left(\mathrm{H}_{2} \mathrm{O}\right)_{n}, \mathrm{n}=8,12,16$, and 20. J. Phys. Chem. 1993, 97, 5208-5210.

(2) Liu, K.; Brown, M. G.; Carter, C.; Saykally, R. J.; Gregory, J. K.; Clary, D. C. Characterization of a Cage Form of the Water Hexamer. Nature, 1996, 381, 501-503.

(3) Wales, D. J.; Hodges, M. P. Global Minima of Water Clusters $\left(\mathrm{H}_{2} \mathrm{O}\right)_{n}, \mathrm{n}<22$, Described by an Empirical Potential. Chem. Phys. Lett. 1998, 286, 65-72.

(4) Nauta, K.; Miller, R. E. Formation of Cyclic Water Hexamer in Liquid Helium: The Smallest Piece of Ice. Science, 2000, 287, 293-295.

(5) Perez, C.; Muckle, M. T.; Zaleski, D. P.; Seifert, N. A.; Temelso, B.; Shields, G. C.; Kisiel, Z.; Pate, B. H. Structures of Cage, Prism, and Book Isomers of Water Hexamer from Broadband Rotational Spectroscopy. Science, 2012, 336, 897-901. 
(6) Wang, Y.; Babin, V.; Bowman, J. M.; Paesani, F. The Water Hexamer: Cage, Prism, or Both. Full Dimensional Quantum Simulations Say Both. J. Am. Chem. Soc., 2012, 134, 1111611119.

(7) Yoo, S.; Aprá, E.; Cheng Zeng, X.; Xantheas, S. S. High-Level Ab Initio Electronic Structure Calculations of Water Clusters $\left(\mathrm{H}_{2} \mathrm{O}\right)_{16}$ and $\left(\mathrm{H}_{2} \mathrm{O}\right)_{17}$ : A New Global Minimum for $\left(\mathrm{H}_{2} \mathrm{O}\right)_{16}$. J. Phys. Chem. Lett., 2010, 1, 3122-3127.

(8) Furtado, J. P.; Rahalkar, A. P.; Shanker, S.; Bandyopadhyay, P.; Gadre, S. R. Facilitating Minima Search for Large Water Clusters at MP2 Level via Molecular Tailoring. J. Phys. Chem. Lett., 2012, 3, 2253-2258.

(9) Do, H.; Besley, N. A. Structural Optimization of Molecular Clusters with Density Functional Theory Combined with Basin Hopping. J. Chem. Phys. 2012, 137, 134106.

(10) Shafer, N.; Bersohn, R. Two and Three Dimensional Laser Induced Fluorescence: Application to Photodissociation of LiI. J. Chem. Phys., 1991, 94, 4817.

(11) Angel, L.; Stace, A. J. Dissociation Patterns of $\left(\mathrm{H}_{2} \mathrm{O}\right)_{n}^{+}$Cluster Ions, for $\mathrm{n}=2-6$. Chem. Phys. Lett., 2001, 345, 277-281.

(12) Kumar, A.; Kolaski, M.; Myoung Lee, H.; Kim, K. S. Photoexcitation and Photoionization Dynamics of Water Photolysis. J. Chem. Phys. A, 2008, 112, 5502-5508.

(13) Garrett, B. C.; Dixon, D.A.; Camaioni, D.M.; Chioman, D.M.; Johnson, M.A.; Jonah, C.D.; Kimmel, M.A.; Miller, J.H.; Rescigno, T.N., Rossky, P.J. at al. Role of Water in ElectronInitiated Processes and Radical Chemistry: Issues and Scientific Advances. Chem. Rev., 2005, $105,355-390$.

(14) Shinohara, H.; Nishi, N.; Washida, N. Photoionization of Water Clusters at $11.83 \mathrm{eV}$ : Observation of Unprotonated Cluster Ions $\left(\mathrm{H}_{2} \mathrm{O}\right)_{n}^{+}(2 \leq \mathrm{n} \leq 10)$ J. Chem. Phys., 1986, 84, 5561. 
(15) Golan, A.; Ahmed, M. Ionization of Water Clusters Mediated by Exciton Energy Transfer from Argon Clusters. J. Phys. Chem. Lett., 2012, 3, 458-462.

(16) Mizuse, K.; Kuo, J. L.; Fujii, A. Structural Trends of Ionized Water Networks: Infrared Spectroscopy of Water Cluster Radical Cations $\left(\mathrm{H}_{2} \mathrm{O}\right)_{n}^{+}(\mathrm{n}=3-11)$ Chem. Sci., 2011, 2, 868876.

(17) Jongma, R. T.; Huang, Y.; Shi, S.; Wodtke, A. M. Rapid Evaporative Cooling Suppresses Fragmentation in Mass Spectrometry: Synthesis of Unprotonated Water Cluster Ions. J. Phys. Chem. A, 1998, 102, 8847-8854.

(18) Barnett, R. N.; Landman, U. Pathways and Dynamics of Dissociation of Ionized $\left(\mathrm{H}_{2} \mathrm{O}\right)_{2}$. J. Phys. Chem., 1995, 99, 17305-17310.

(19) Barnett, R. N.; Landman, U. Structure and Energetics of Ionized Water Clusters: $\left(\mathrm{H}_{2} \mathrm{O}\right)_{n}^{+}, \mathrm{n}$ = 2-5 J. Phys. Chem. A, 1997, 101, 164-169.

(20) Liu, H. T.; Muller, J. P.; Beutler, M.; Ghotbi, M.; Noack, F.; Radloff, W.; Zhavoronkov, N.; Schulz, C. P.; Hertel, I. V. Ultrafast Photo-Excitation Dynamics in Isolated, Neutral Water Clusters. J. Chem. Phys., 2011, 134, 094305.

(21) Gill, P. M. W.; Radom, L. Structures and Stabilities of Singly Charged Three-Electron Hemibonded Systems and Their Hydrogen-Bonded Isomers. J. Am. Chem. Soc., 1988, 110, 49314941.

(22) Yamaguchi, S.; Kudoh, S.; Kawai, Y.; Okada, Y.; Orii, T.; Takeuchi, K. Collisional Reaction of Water Cluster Cations $\left(\mathrm{H}_{2} \mathrm{O}\right)_{n}^{+}\left(\mathrm{n}=2\right.$ and 3) with $\mathrm{D}_{2} \mathrm{O}$ Chem. Phys. Lett., 2003, 377, 37-42.

(23) Lee, H. M.; Kim, K. S. Water Dimer Cation: Density Functional Theory vs Ab Initio Theory. J. Chem. Theory Comput., 2009, 5, 976-981.

(24) Lee, H. M.; Kim, K. S. Water Trimer Cation. Theor. Chem. Acc., 2011, 130, 543-548. 
(25) Livshits, E.; Granot, R. S.; Baer, R. A Density Functional Theory for Studying Ionization Processes in Water Clusters. J. Phys. Chem. A, 2011, 115, 5735-5744.

(26) Chipman, D. M. Hemibonding between Hydroxyl Radical and Water. J. Phys. Chem. A, 2011, $115,1161-1171$.

(27) Pieniazek, P. A.; Vande Vondele, J.; Jungwirth, P.; Krylov, A. I.; Bradforth, S. E. Electronic Structure of the Water Dimer Cation. J. Phys. Chem. A, 2008, 112, 6159-6170.

(28) Cheng, Q.; Evangelista, F.A.; Simmonett, A. C.; Yamaguchi, Y.; Schaefer III, H. F. Water Dimer Radical Cation: Structures, Vibrational Frequencies, and Energetics. J. Phys. Chem. A, 2009, 113, 13779-13789.

(29) Wales, D. J.; Doye, J. P. K. Global Optimization by Basin-Hopping and the Lowest Energy Structures of Lennard-Jones Clusters Containing up to 110 Atoms. J. Phys. Chem. A, 1997, $101,5111-5116$.

(30) Hodges, M.P.; Wales, D. J. GLobal Minima of Protonated Water Clusters. Chem. Phys. Lett., 2000, 324, 279.

(31) James, T.; Wales, D. J. Protonated Water Clusters Described by an Empirical Valence Bond Potentials. J. Chem. Phys., 2005, 122, 134306.

(32) Shao, Y.; Molnar, L. F.; Jung, Y.; Kussmann, J.; Ochsenfeld, C.; Brown, S. T.; Gilbert, A. T. B.; Slipchenko, L. V.; Levchenko, S. V.; O’Neill, D. P. at al. Advances in Methods and Algorithms in a Modern Quantum Chemistry Program Package. Phys. Chem. Chem. Phys., 2006, $8,3172-3191$.

(33) Iwamatsu, M.; Okabe, Y. Basin Hopping with Occasional Jumping. Chem. Phys. Lett., 2004, 399, 396-400.

(34) Becke, A. D. Density-Functional Thermochemistry. III. The Role of Exact Exchange. $J$. Chem. Phys., 1993, 98, 5648. 
(35) Stephens, P. J.; Devlin, F. J.; Chabalowski, C. F.; Frisch, M. J. Ab Initio Calculation of Vibrational Absorption and Circular Dichroism Spectra Using Density Functional Force Fields. $J$. Phys. Chem., 1994, 98, 11623-11627.

(36) Grimme, S. Semiempirical GGA-type density functional constructed with a long-range dispersion correction J. Comput. Chem., 2006, 27, 1787-1799.

(37) MOLPRO, version 2006.1, a package of ab initio programs, Werner, H.-J.; Knowles, P.J.; Lindh, R.; Manby, F.R., Schütz; M., Celani, P.; Korona, T.; Rauhut, G.; Amos, R.D.; Bernhardsson, A. at al.

(38) Andersson, M. P.; Uvdal, P. New Scale Factors for Harmonic Vibrational Frequencies Using the B3LYP Density Functional Method with the Triple- $\zeta$ Basis Set 6-311+G(d,p) J. Phys. Chem. A, 2005, 109, 2937-2941.

(39) Chipman, D. M. Absorption Spectrum of OH Radical in Water. J. Phys. Chem. A, 2008, 112, 13372-13381. 
Figure 1: Illustrative structures and associated spin densities for the different structural motifs observed. a: a cluster with a hemibond between two oxygen atoms, b: a cluster with a directly bonded $\mathrm{H}_{3} \mathrm{O}^{+}$ion and $\mathrm{OH}$ radical, c: a cluster with a non-directly bonded $\mathrm{H}_{3} \mathrm{O}^{+}$ion and $\mathrm{OH}$ radical, d: a cluster with an $\mathrm{OH}$ radical hemibonded with a water molecule. Regions of increasingly high spin density are represented by yellow, green and blue.

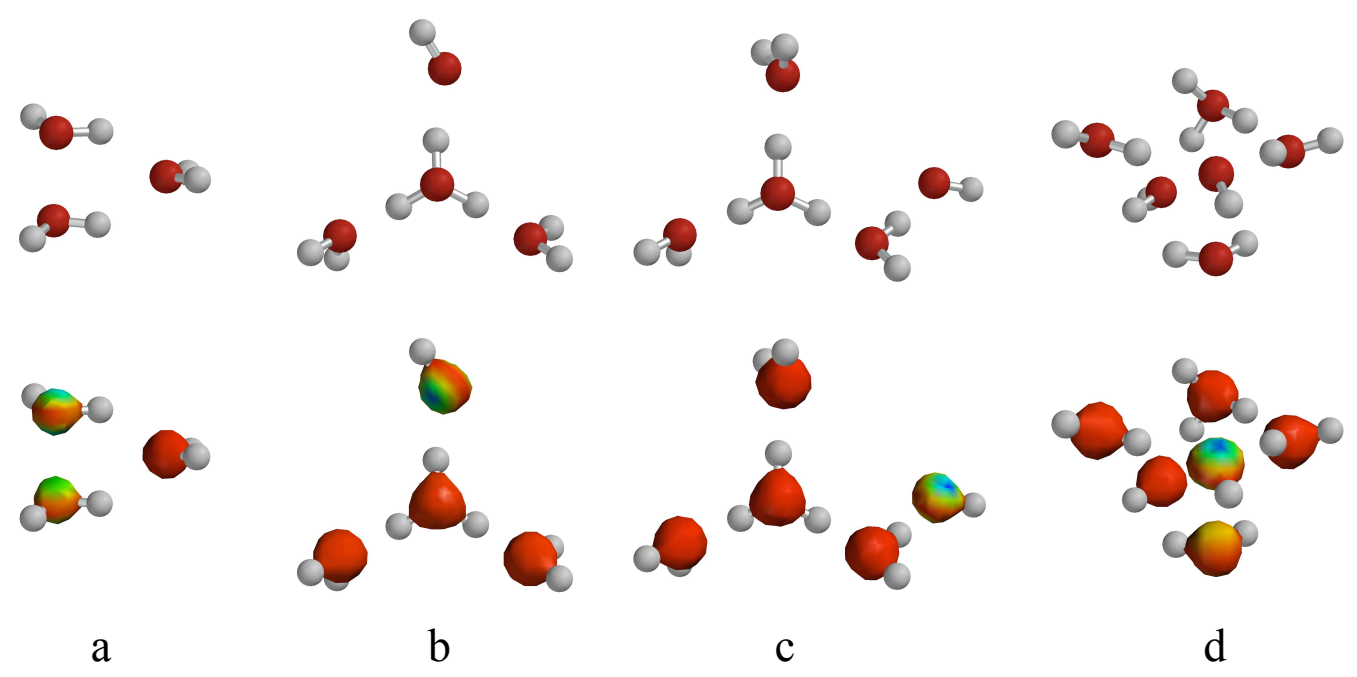


Figure 2: The oxygen-oxygen $\sigma$ and $\sigma^{*}$ orbitals involved in the hemibonding between two water molecules in the $\left(\mathrm{H}_{2} \mathrm{O}\right)_{3}^{+}$cluster.

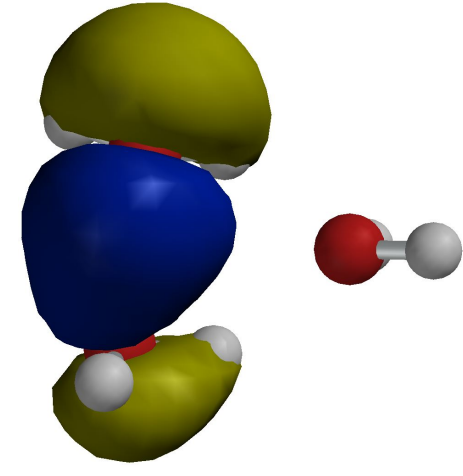

$\sigma$

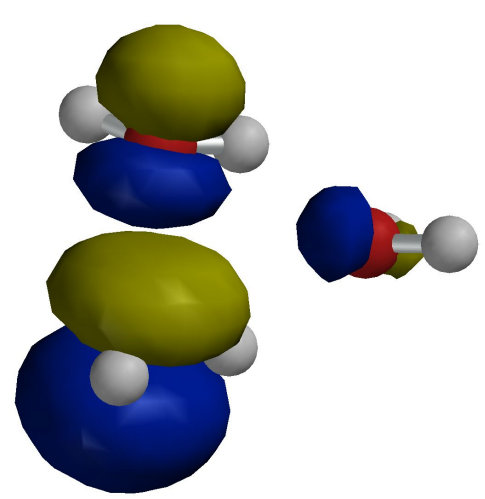

б* 
Figure 3: Structures and relative energies $\left(\Delta E_{e}\right)$ of the low energy isomers of the ground state of the $\left(\mathrm{H}_{2} \mathrm{O}\right)_{n=3-6}^{+}$clusters. Relative energies including zero point energies $\left(\Delta E_{0}\right)$ shown in parenthesis. Hydrogen bonds are denoted by black dotted lines and hemibonds are shown by blue dotted lines.
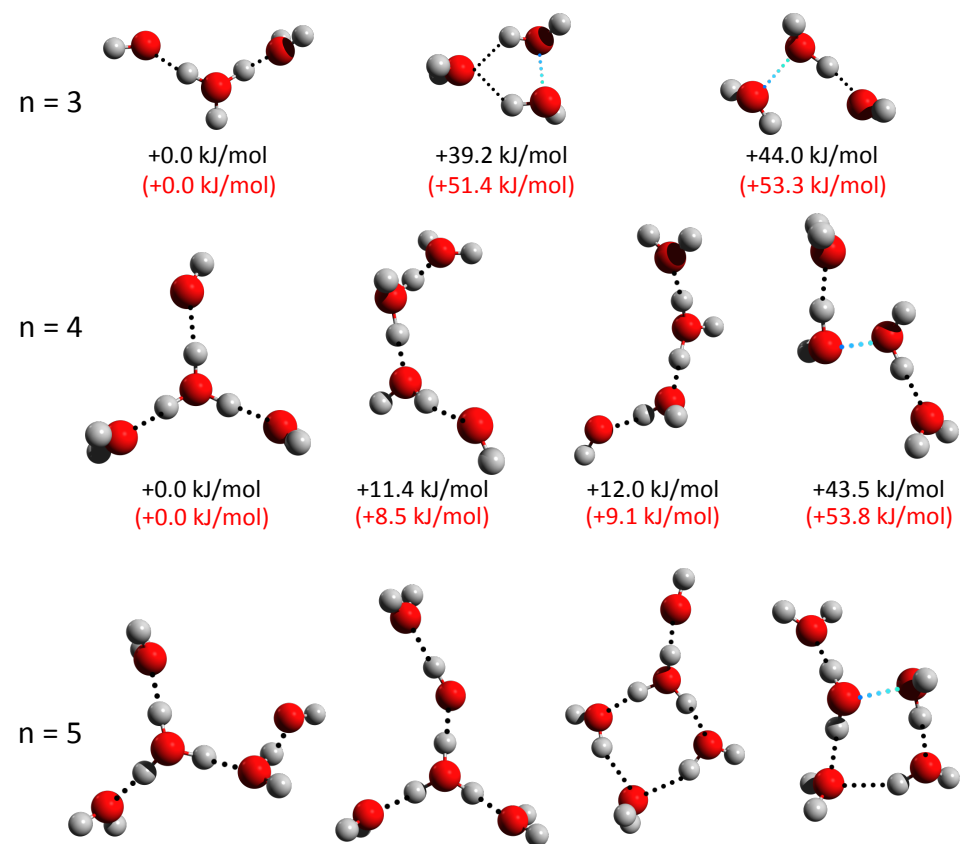

$+0.0 \mathrm{~kJ} / \mathrm{mol}$ $(+0.0 \mathrm{~kJ} / \mathrm{mol})$
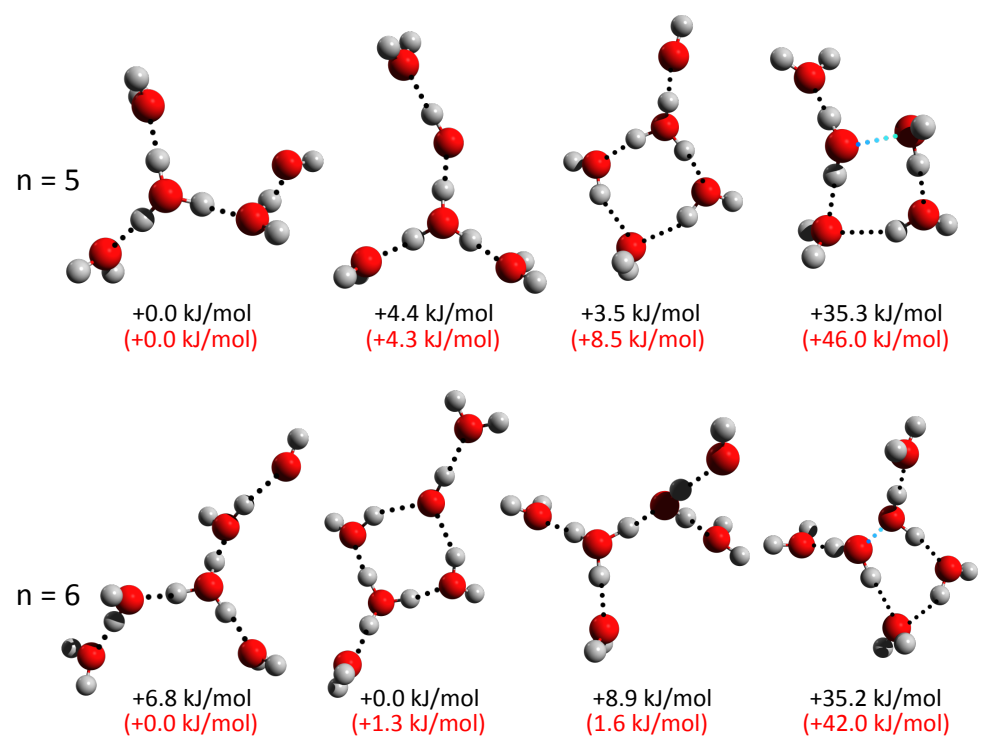
Figure 4: Structures and relative energies $\left(\Delta E_{e}\right)$ of the low energy isomers of the ground state of the $\left(\mathrm{H}_{2} \mathrm{O}\right)_{n=7-9}^{+}$clusters. Relative energies including zero point energies $\left(\Delta E_{0}\right)$ shown in parenthesis. Hydrogen bonds are denoted by black dotted lines and hemibonds are shown by blue dotted lines.
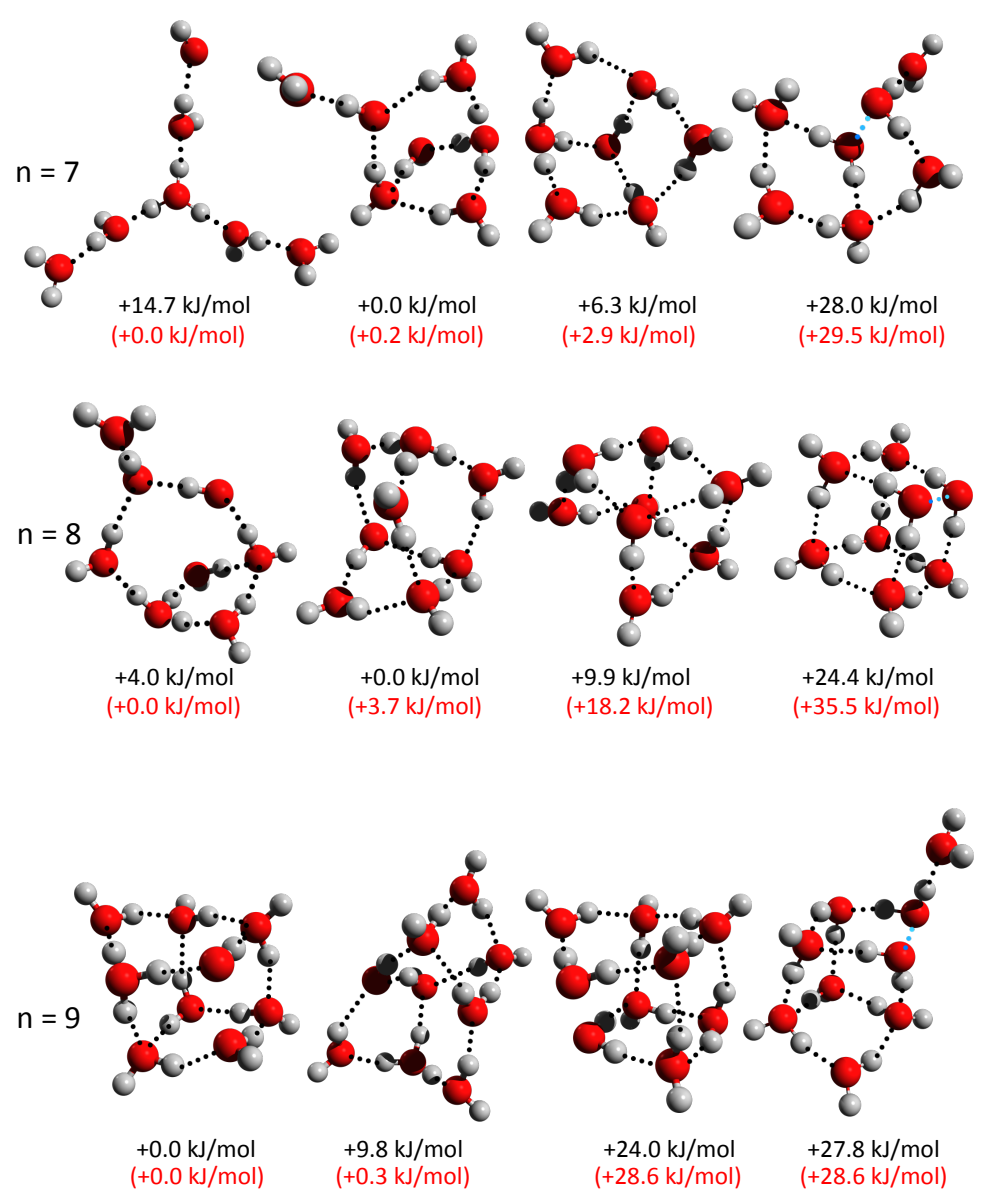
Figure 5: Comparison of the computed IR spectra of the two lowest energy isomers with experiment. Blue (lower) lines - computed spectra for the lowest energy isomers. Red (middle) lines computed spectra for the second lowest energy isomer. Black (upper) lines - experimental spectra from the work of Mizuse et al., reference 16. For $n=9$, the Boltzmann weighted spectrum for the two lowest energy isomers is shown in green.
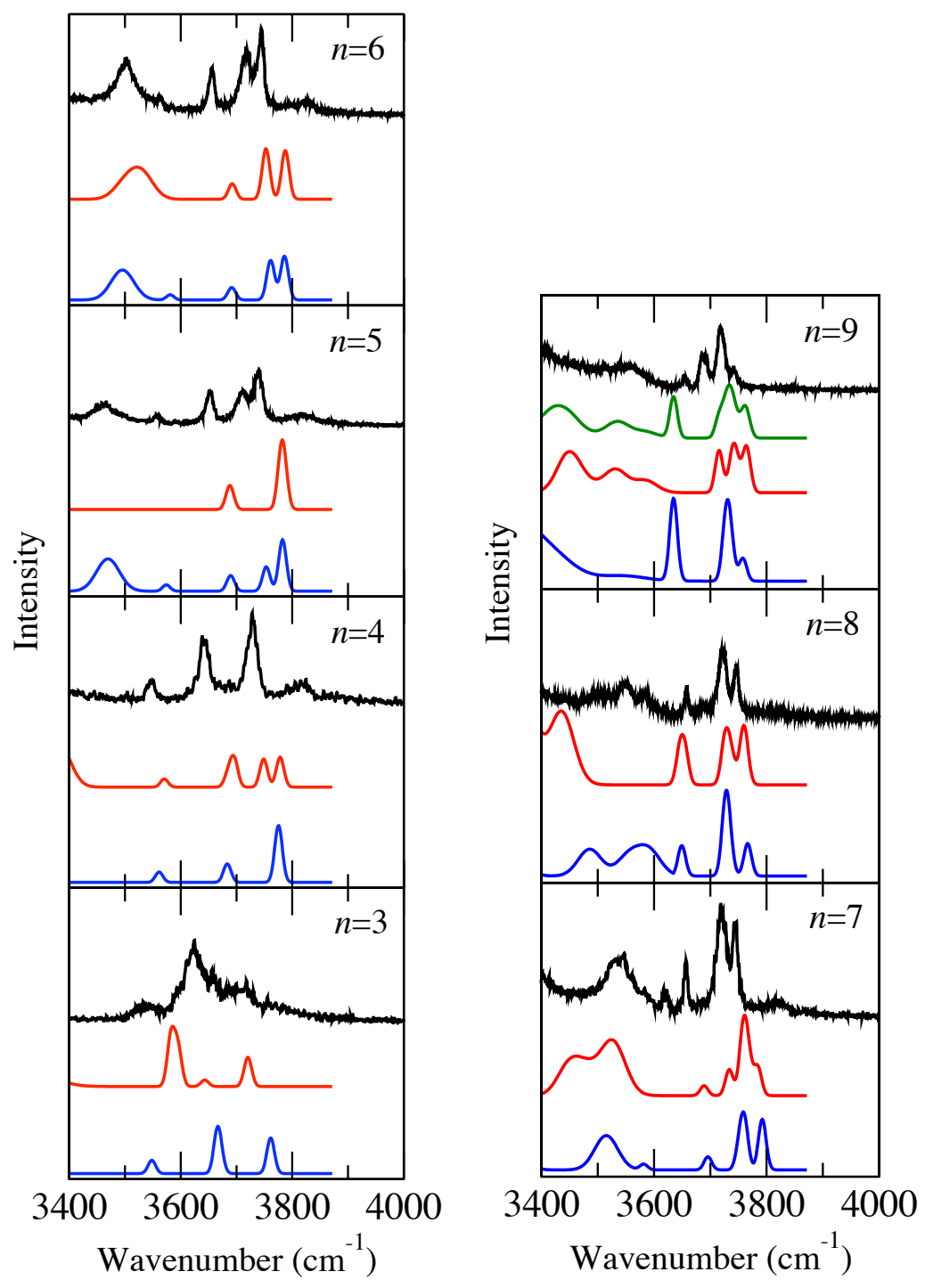
Table of Contents Graphic

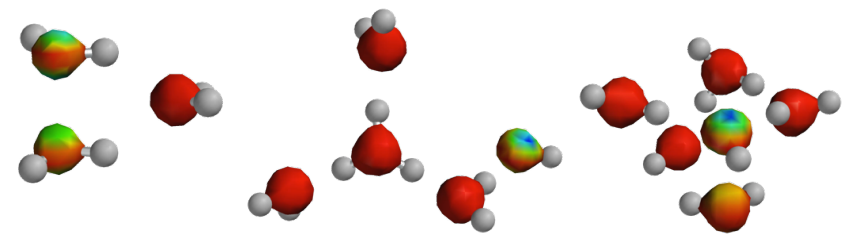

